\title{
Trayectorias Educativas en el Marco de la Implementación del Ingreso Irrestricto en una Universidad Argentina
}

\section{Educational Trajectories in the Context of the Implementation of Unrestricted Admission to an Argentine University}

\author{
Gloria González ${ }^{1}$ * \\ Florencia Nogueira ${ }^{1}$ \\ Macarena del Valle ${ }^{1,2}$ \\ Carolina Grossi ${ }^{1}$ \\ ${ }^{1}$ Universidad Nacional de Mar del Plata, Argentina \\ 2 CONICET, Instituto de Psicología Básica, Aplicada y Tecnología, Argentina
}

\begin{abstract}
En las últimas décadas, las políticas de democratización de la educación superior llevaron a la modificación de las normativas de ingreso y permanencia a las universidades nacionales argentinas. Esta investigación tiene por objetivo explorar las percepciones de los estudiantes universitarios sobre sus propias trayectorias educativas en el marco de la implementación de las políticas de ingreso irrestricto, y en relación con factores sociales, económicos e institucionales. Se realizan entrevistas semi-estructuradas a 40 estudiantes. Los resultados indican una divergencia en las trayectorias educativas a favor de quienes pertenecen a sectores sociales más favorecidos, quienes pueden ser sostenidos económicamente por sus padres y quienes no necesitan de salir a trabajar. También surgen en las entrevistas comentarios sobre las dificultades que afronta el sistema universitario (infraestructura, recursos humanos) para responder a las demandas que conlleva el incremento en el número de estudiantes. En general, se valoriza la importancia de la formación y apoyo al ingresante, y la necesidad de docentes con una alta formación académica, pedagógica y profesional.
\end{abstract}

Descriptores: Democracia; Acceso; Educación superior; Justicia social; Recursos.

In the last decades, public policies aimed at the democratization of education have led to the modification of the regulations for admission and retention in the national universities of Argentina. This research aims to explore the university students' perceptions about their own educational trajectories in the context of the unrestricted entry policies, and in relation to social, economic, and institutional factors. Semi-structured interviews were conducted with 40 students. The results indicate a divergence in educational trajectories in favor of the groups of students who belong to the most favored social sectors; those who can be economically supported by their parents and those who do not need to work. The interviews also reflect the difficulties that the university system faces (infrastructure, human resources) in responding to the demands of the increase in the number of students. In general, students value the importance of training and supporting freshmen, and the need for teachers with a high academic, pedagogical, and professional background.

Keywords: Democracy; Access; Higher education; Social justice; Resources.

*Contacto: gigon@mdp.edu.ar

ISSN: 2254-3139

www.rinace.net/riejs/

revistas.uam.es/riejs
Recibido: $\quad 13$ de marzo 2020

$1^{\text {a }}$ Evaluación: 1 de junio 2020

$2^{\text {a }}$ Evaluación: 9 de septiembre 2020

Aceptado: 2 de octubre 2020 


\section{Introducción}

En distintos países de América Latina ha acontecido un fenómeno de ampliación de compromisos y metas educativas, aunque las modalidades de implementación no han estado exentas de una notoria y marcada desigualdad (Ezcurra, 2019). Argentina, así como muchos otros países, ha atestiguado un aumento exponencial en las matriculaciones universitarias, fenómeno que ha dado en llamarse masificación de la educación superior (López Segrera, 2016). Sin embargo, considerando que esta expansión ha tenido lugar en un contexto histórico específico (desplazamiento del denominado Estado de Bienestar, reorientación al neoliberalismo y fenómenos sociales asociados), las metas educativas previstas se advierten lejos de ser alcanzadas. Así, en Argentina, el éxito en la ampliación de la matrícula no se ha reflejado en la tasa de graduación (González et al., 2020): de los alumnos que se inscriben en las universidades nacionales de nuestro país, solo egresa aproximadamente el 22\% (García de Fanelli, 2014).

De este modo, el aumento en las tasas de matriculación por sí solo no representa garantías en lo que atañe a la permanencia y al egreso de los estudiantes. Por el contrario, el escenario se asemeja más bien a lo que Ezcurra (2011) llama inclusión excluyente, es decir, aunque el número de beneficiarios haya aumentado, el sistema sigue sin poder evitar la expulsión de una gran cantidad de elementos, principalmente aquellos que provienen de sectores sociales desfavorecidos (Coschiza et al., 2016). En palabras de Tinto (2006, 2012), las políticas de ingreso se transforman en puertas giratorias, por las que muchos jóvenes alumnos acceden a un mundo previamente impensado, pero en el que el abandono y la interrupción de la trayectoria universitaria los afecta en gran medida. Por eso, el interés del presente estudio es explorar las percepciones de los estudiantes universitarios sobre sus propias trayectorias educativas en el marco de la implementación de las políticas de ingreso irrestricto, y en relación con factores sociales, económicos e institucionales.

\section{Revisión de la literatura}

\subsection{Masificación de la educación superior en América Latina}

Tradicionalmente, los estudios universitarios se caracterizaron por un estricto carácter elitista (Brunner, 2012). En Argentina, aunque la educación superior pública es completamente gratuita desde hace más de 70 años, tanto en términos de matriculación como de cuotas o tasas, los ingresos a las universidades se encontraban restringidos por exámenes o dispositivos semejantes. Estos mecanismos restrictivos impedían el acceso de estudiantes con menores recursos socioeconómicos, funcionando como una selección social (Chiroleu, 2009a; Mollis, 2008). Esto fue naturalizado hasta que, a comienzos de los años ochenta, los crecientes discursos de democratización política en Latinoamérica dieron lugar a la masificación de la educación superior, es decir al aumento exponencial en las matriculaciones universitarias (Rama, 2006). En muchos casos, como supuesta garantía de la igualdad de oportunidades, se implementaron sistemas de ingreso directo o irrestricto (Parrino, 2010). Estos, a diferencias de los sistemas restrictivos que exigen la aprobación de un examen de ingreso, no poseen mecanismos eliminatorios y se acepta a todos los estudiantes (aunque pueden, o no, ofrecerse cursos de ingreso, obligatorios u optativos).

Sin embargo, a pesar de la gran ampliación de la matrícula, una enorme proporción de estudiantes sigue abandonando sus estudios (González et al., 2020). Las inquietudes respecto a la relación entre la masificación y la democratización de la educación superior 
son equiparables a las diferencias entre "ingreso" y "acceso". En este sentido, ingreso supone la condición de matricularse como estudiante universitario, pero acceso se asocia a la apropiación real del perfil estudiantil y, por tanto, a toda una serie de factores relacionados con las características socioeconómicas del ingresante (Rabossi, 2014). Si bien la idea de igualdad es lo que orienta la implementación del ingreso irrestricto, el ingresante se verá rápidamente enfrentado a un escenario en el que no podrá desempeñarse exitosamente (Rawls, 1971).

Al respecto, Argentina tiene un mayor número de estudiantes que otros países de la región como Chile o Brasil, pero mayor deserción y, por lo tanto, menos graduados (Guadagni, Lima y Boero, 2019). Rabossi (2014) argumenta que la baja tasa de graduación argentina es consecuencia directa de la gratuidad del sistema universitario y de la falta de exámenes de admisión. En cambio, según el autor, en Chile o Brasil, las tasas de graduación son mayores justamente por la existencia de exámenes como el Vestibular (Brasil) o la Prueba Nacional (Chile). Además, Brasil ofrece educación universitaria gratuita, pero limita el cupo de admisiones, lo cual no ocurre en Argentina. De todos modos, tal como sugiere Latapí Sarre (2012), un aspecto fundamentalmente cualitativo como la justicia social, no puede reflejarse meramente en indicadores cuantitativos como tasas o proporciones. Aunque la gratuidad del sistema y la igualdad de acceso supongan condiciones de posibilidad (Bolívar, 2012), lo que ocurre al interior de cada trayectoria educativa también necesita ser explorado para alcanzar un orden social justo.

\subsection{El problema del acceso real y la democratización}

Fernández Lamarra y Costa de Paula (2002) exploran el fenómeno del acceso y permanencia a las universidades y lo interpreta desde dos frentes obstaculizadores: por un lado, desde las dificultades del estudiantado para adaptarse a las instituciones de educación superior; por el otro, desde las instituciones para crear mecanismos de acceso, recepción y permanencia. Así, parecería, que el encuadre organizacional universitario desconoce aún que sus aulas son habitadas, a partir del proceso de masificación, por contingentes de estudiantes antes excluidos, con posiciones en desventaja en las distintas formas de distribución del capital (Bordieu, 2005). En este sentido, la masificación produce cambios en el perfil social del estudiantado, incorporando ahora estudiantes del interior de los países, estudiantes part-time, estudiantes que trabajan, entre otros (Rama, 2009).

Así, aunque las políticas gubernamentales y el creciente discurso social conlleven un mandato de inclusión, esto confronta con una estructura pedagógica tradicional poco flexible (Di Piero y Mataluna, 2018; González y del Valle, 2020; Ziegler, 2014). También otros autores (p. ej., Carlino, 2004; Ezcurra, 2011) sugieren que existen ciertas dimensiones ligadas al saber estudiar (como por ejemplo la capacidad de comprensión lectora y de expresión escrita) que poseen relevantes implicancias en las actividades académicas, y en la planificación y organización del tiempo. Esto hace a lo que se ha dado en llamar el oficio de ser alumno (Perrenoud, 2006), oficio que se aprende para poder dar respuesta a las expectativas docentes e institucionales que en muchos casos no son explícitas. En este sentido, para muchos alumnos provenientes de sectores sociales desfavorecidos, se trata de la primera generación en acceder a la educación universitaria, y hacen lo posible por tratar de incorporarse a un nivel académico con códigos propios que les provoca extrañamiento.

Otros autores (Ezcurra, 2019; Mataluna, 2018) abordan la cuestión de la deserción como posible efecto colateral del ingreso masivo, y consideran entre las tendencias estructurales 
globales a la situación de desventaja socioeconómica como uno de los condicionantes de mayor impacto. Así, según Chiroleu (2009a, p. 6), esta situación no es suficientemente reconocida en Argentina, y los proyectos de inclusión educativa en la Educación Superior se escudan en:

"la primacía de valores como la excelencia y el mérito, componentes indisociables de la educación superior, que se asocia contemporáneamente con lógicas excluyentes. Focalizando en el individuo se considera que las personas son premiadas por sus acciones y sus logros los cuales se ligan al esfuerzo, la perseverancia y el trabajo constante. Desde esta perspectiva se sostiene que, independientemente de su origen y situación inicial, cualquier persona dispuesta a someterse a ese esfuerzo alcanzaría las mismas metas".

La igualdad en el ingreso, no elimina las disparidades iniciales (Morduchowicz, 2003) y aunque en algunos países se hayan solucionado los problemas del ingreso de nuevos estudiantes, el foco se traslada ahora al trayecto educativo más general, a las vivencias particulares y al rendimiento académico (Cuenca, 2012). En Uruguay, Fiori y Ramírez (2013) aseguran que la deserción académica es una de los factores que más incide en la accesibilidad y cobertura de la educación superior, y que existen aspectos académicos, institucionales y sociodemográficos que inciden sobre este fenómeno. Por ejemplo, los autores reportan que las mujeres tienen menos riesgo de deserción que los hombres, y que, mientras mayor es la edad de ingreso, mayor es la posibilidad de abandonar los estudios. También las personas solteras y sin hijos son quienes más permanencia presentan, al igual que quienes no trabajan (asociado esto a que reciben sostén económico de sus familias de origen).

En México, Mendoza y Flores (2007) indican que los estudiantes desertan menos cuando son acompañados en su trayectoria educativa por medio de dispositivos de apoyo y docentes capacitados para tal fin. Otros autores (p. ej., Márquez García y Padua Arcos, 2009) han reportado la importancia de la cercanía y el afecto de los docentes, aunque ciertos estudios (p. ej., Aisenson et al., 2011; Klein, 2011) indican que no todos los estudiantes refieren tener buenas relaciones con los docentes; de hecho, la distribución del grado de satisfacción con el vínculo docente suele ser muy variada. También el apoyo de los pares ha sido sugerido como una herramienta muy valiosa en las trayectorias educativas (Cardozo-Ortiz, 2011).

\subsection{El caso de una universidad nacional Argentina}

En Argentina, el debate sobre la admisión a las universidades públicas y los mecanismos de exclusión/inclusión de la educación superior se encontraban vigentes desde hace ya varios años (Chiroleu, 2009b; Porto et al., 2004). En el año 2013, comienza a discutirse en las entidades gubernamentales la problemática de la democratización del ingreso y en el año 2015 (entre otras consideraciones basadas en el derecho a la educación), el resultado se materializa en la modificación de la Ley de Educación Superior, eliminando los exámenes restrictivos de ingreso y cualquier mecanismo de exclusión semejante.

En este escenario, ya en el año 2014, en la Facultad de Psicología de una Universidad Nacional de la provincia de Buenos Aires, se decide la eliminación del examen de ingreso de carácter restrictivo. La modalidad eliminatoria vigente hasta 2014 suponía rendir un examen eliminatorio, lo cual podía hacerse luego asistir a un curso de ingreso nivelatorio o de forma libre, sin curso de ingreso. La nueva modalidad, de ingreso irrestricto, consiste en un Proyecto de Ingreso (curso de ingreso obligatorio no eliminatorio) organizado en 
torno a dos espacios: uno de clases de formación sobre la Psicología y la Universidad y otro de talleres vocacionales. Luego de esta modificación, la cantidad de ingresantes a la Facultad aumentó en aproximadamente 40\%, debiendo la institución responder a una demanda de casi 1000 estudiantes, cuando los promedios de años previos rondaban los 500 .

En el nuevo Proyecto de Ingreso, las clases de formación desarrollan actividades teóricoprácticas individuales y grupales para los ingresantes, con textos cuya complejidad es similar a los del inicio de la carrera. También se desarrollan trabajos prácticos individuales y tutorías personalizadas, con los que se pretende poner a disposición del aspirante aquellas estrategias, competencias y habilidades que necesitará para aprehender los contenidos académicos. A su vez, se transmiten las normativas en las que estará encuadrado por su condición de estudiante, y se propicia el proceso de enculturación (Carlino, 2005), que va más allá de la alfabetización académica y que es necesario para su inclusión en la Universidad.

Respecto del espacio de taller vocacional, el mismo es de reflexión-acción y versa sobre la construcción del rol de estudiante universitario y del profesional psicólogo. Incluye no solamente aspectos formativos sino de orientación vocacional, individuales y grupales. En el mismo el docente-orientador colabora con el aspirante en la reflexión acerca su proceso de decisión, y sobre las representaciones que tiene acerca de ser estudiante universitario y del ejercicio de la Psicología como actividad científico-profesional.

Así, el acceso es irrestricto, pero se encuentra acompañado de instancias previas al cursado de primer año que favorezcan la posibilidad concreta de acceso y permanencia en la Facultad de Psicología. El proceso de acceso a una carrera universitaria es complejo. No consiste meramente en matricularse y llevar a cabo una serie de trámites administrativos, sino en aprehender las formas que llevan a ser estudiantes. Por tanto, para este estudio, se optó por la utilización del constructo trayectorias educativas, puesto que el mismo permite aunar, en una misma categoría, las elecciones y experiencias de los sujetos, las experiencias familiares, la posible existencia y disponibilidad de propuestas institucionales y el contex to social que opera condicionando los recorridos y las prácticas (Montes y Sendón, 2006). Este concepto enlaza las prácticas y las estrategias desplegadas por los actores y también los elementos estructurales de los grupos sociales y de quienes son puestos en acción en un determinado contexto, entre ellos los capitales culturales, económicos y simbólicos (Tiramonti y Ziegler, 2008).

Por todo lo antedicho, el objetivo de este estudio es explorar las percepciones de los estudiantes universitarios sobre sus propias trayectorias educativas en el marco de la implementación de las políticas de ingreso irrestricto, y en relación con factores sociales, económicos e institucionales. En particular, se espera poder indagar en las perspectivas idiosincráticas de la propia trayectoria educativa, en relación con los dispositivos institucionales, el funcionamiento de la universidad y los vínculos establecidos en ella. También se espera explorar estas experiencias educativas poniéndolas en perspectiva con ciertos aspectos personales (demográficos y socioeconómicos), como el género, el nivel educativo de la familia de origen y la condición laboral. 


\section{Método}

La metodología de trabajo es cualitativa, y el estudio es de tipo exploratorio-descriptivo. Para la recolección de datos, se construye instrumento ad hoc que consiste en un cuestionario con preguntas cerradas y una entrevista semiestructurada. En el cuestionario se indaga acerca de algunos aspectos sociodemográficos: los datos personales, el año de ingreso a la carrera, el lugar de residencia, la condición laboral, los familiares a cargo y el máximo nivel de educación alcanzado en su grupo familiar de origen. En la entrevista se indaga acerca del impacto de las modalidades de ingreso irrestricto sobre el acceso, permanencia y regularidad en la trayectoria educativa, las disponibilidades económicas y las dificultades enfrentadas, el acompañamiento durante el primer año de la carrera y su experiencia de acceso al sistema, la perspectiva vocacional, los aspectos institucionales que cree que presentan falencias y los vínculos humanos establecidos desde su llegada a la universidad. Se opta por la metodología de entrevista con el propósito de analizar en un único encuentro personal con el estudiante, los motivos subyacentes a las diferentes decisiones de cada trayectoria educativa.

En primer término, se selecciona mediante un muestreo aleatorio simple una muestra de cada cohorte (últimos estudiantes en ingresar por examen en el año 2014, y primeros estudiantes en ingresar sin examen en el año 2015) y se los invita por mail a concurrir a una entrevista en la Facultad, anticipándole que se trata de una investigación sobre trayectorias académicas. Frente a una baja tasa de respuestas por mail se realiza posteriormente un contacto telefónico, lo que incide muy positivamente en la aceptación a concurrir a la entrevista. Se realizan 28 entrevistas con estudiantes activos y 12 entrevistas (más breves) a ingresantes que abandonaron sus estudios tempranamente (primer año) pero acceden a ser entrevistados. Del total de los entrevistados, ocho son de género masculino y 32 de género femenino. Esta distribución de mayoría de personas de género femenino es común en las carreras de Psicología de las universidades nacionales argentinas, y, en particular, en la facultad en la que se realizó el estudio el $84 \%$ de las personas egresadas son de sexo femenino (Secretaría de Políticas Universitarias, 2020). Llegado el punto donde se saturan las categorías teóricas se da por cumplimentado el proceso de entrevistas a realizar.

Para el análisis de los datos, se consideran las siguientes categorías de análisis a) Recursos económicos y factores sociodemográficos b) Adaptación al funcionamiento institucional, c) Infraestructura y equipamiento disponible, d) Funcionamiento institucional y diseño curricular, e) Vínculos pedagógicos, y f) Grupo de pares. Además, el interior de las categorías, los resultados se ponen en perspectiva en función del género, el nivel educativo de la familia de origen y la condición laboral personal. Las categorías seleccionadas surgen en parte de la revisión de la literatura, y en parte del análisis mismo de los datos.

\section{Resultados}

\subsection{Recursos económicos y factores sociodemográficos}

Las dificultades económicas y la necesidad de ingresar tempranamente al mercado laboral aparecen como motivo en el abandono de los estudios o, en el mejor de los casos, en el rezago. Así nos lo indica un estudiante varón (E-29), que deja la carrera en segundo año para salir a trabajar luego del fallecimiento del padre. Asimismo, el siguiente es un 
fragmento de la entrevista de un estudiante que ingresó en el año 2015 y es la primera generación de universitarios en su familia. Como se ve obligado a trabajar, abandona durante su primer año. Decide cambiarse a una carrera más breve (Profesorado en Educación Física) y espera, una vez terminada esta, poder retomar Psicología:

Veía muy lejos en tiempo la salida laboral. No sabía si iba a poder estar sin trabajar cinco años (...) evalué las distancias por el gasto en transporte. (E-23)

También aparecen discursos similares en personas que estudian y trabajan simultáneamente:

Es un sacrifico económico (...) pude una vez que accedí a las becas, si no, no podía comprar el material (...) estudio de a ratos y así organizo las prioridades del día. (E1; estudiante de 32 años, trabaja parcialmente, segunda generación universitarios; se encuentra haciendo materias de $4^{\circ}$ año)

Limita mucho el trabajar en relación de dependencia. (E-2; estudiante, 43 años, a cargo de su padre, trabaja 4 horas al día, primera generación universitarios; se encuentra haciendo materia de $3^{\circ}$ año)

"Debería sacarse el requisito de obligatoriedad de todas las clases porque es difícil para un estudiante que trabaja poder acomodar los horarios (...). (E-19; trabaja 4 horas diarias; primera generación de universitarios; se encuentra haciendo materias de $3^{\circ}$ año)

Aquellos estudiantes jóvenes que no necesitan trabajar porque son sostenidos económicamente por su familia, reconocen la difícil situación de quienes trabajan y estudian simultáneamente, y lo identifican como un obstáculo para el desempeño. El siguiente fragmento es de un estudiante de 21 años cuyos padres son ambos universitarios:

Nunca pensé en trabajar (...) en mi familia se concibe a la Universidad como prioridad (...) los que trabajan se atrasan (...) dedicarme exclusivamente al estudio es lo que hizo que tuviera un buen desempeño. (E-7; no trabaja; se encuentra haciendo materias de 5 año)

En términos generales, se hallan coincidencias entre las entrevistas al considerar que existe una baja probabilidad de mantener al día la carrera universitaria si se trabaja. Los tiempos institucionales (en este caso, cinco años) les resultan "largos". Esta apreciación la realizan no sólo quienes han experimentado un rezago en sus trayectorias o quienes no pudieron mantenerse dentro del sistema, sino también los alumnos regulares que mantienen un buen desempeño. Esto es advertido tanto por quienes dieron examen de ingreso como por quienes accedieron de modo directo.

Unas pocas de las personas entrevistadas eran mujeres con hijos (no hubo hombres con hijos entre los entrevistados). En esos casos, también destacan la dificultad que supone hacer coincidir los tiempos familiares con los institucionales. También suelen mencionar que es un problema la cantidad de carga horaria presencial obligatoria que tiene la carrera.

Me la paso haciendo mil malabares con la organización de mi familia (...) se me complica para venir a todas las clases. (E-25; 33 años, 4 hijos, no trabaja, primera generación de universitarios; se encuentra haciendo materia de $3^{\circ}$ año)

Es increíble la cantidad de horas que hay que ir [a clase]... yo propondría que se aumente la cantidad de faltas permitidas y que haya más oferta de horarios.... (E17; es docente y madre; tiene un profesorado en Filosofía, y ha dejado la carrera de Psicología en suspenso por ahora, con solo el 20\% de las materias aprobadas) 
Hice dos años y medio, pero después dejé porque tuve a mis hijos y tenía que ocuparme de ellos. (E-32; 35 años; primera generación de universitarios; abandonó la carrera luego de dos años)

\subsection{Adaptacion al funcionamiento institucional}

En la mayoría de los casos, es altamente valorada la existencia de un curso de acompañamiento al ingreso o modalidades similares de aproximación a la vida universitaria. Hay consenso en la idea de que una instancia pre-universitaria (“curso”) es útil de diferentes formas.

Me sirvió mucho el examen de ingreso. Aprendí un montón. Demuestra lo que es estar
dentro de la universidad (...) cuando no sabía dónde ir, me decían en el centro de
estudiantes. (E-1 1; 24 años; abandona luego de dos años. Ingresa a Medicina,
abandona nuevamente, e ingresa a la carrera terciaria de Enfermería en una
institución privada; no trabaja; se mantiene con apoyo familiar; primera
generación de universitarios) El curso de ingreso me sirvió para orientarme en la universidad. (...) Es necesario un asesoramiento a lo largo del $1^{\circ}$ año. (E-13; 28 años, trabaja, primera generación universitarios; se encuentra realizando materias de $3^{\circ}$ año)

El primer año es crítico, y eso que hice curso de ingreso que me sirvió me sirvió motivacionalmente. (E-5; 21 años; no trabaja; un padre con estudios universitarios incompletos; se encuentra realizando materias de $5^{\circ}$ año)

El curso hace que el contacto con la universidad sea menos violento. (E-18; 22 años; no trabaja; padres universitarios; se encuentra realizando materias de 3 año)

En particular, lo destacan como un recurso que podría disminuir la brecha entre educación secundaria y la educación universitaria, tanto desde la perspectiva de orientación vocacional, como de acercamiento al campo disciplinar. La inserción al funcionamiento institucional universitario es descripta como un "salto", y no como una prosecución del recorrido educativo en sus diferentes etapas (secundaria-universitaria).

La escuela secundaria y la Universidad son dos mundos aparte. Acá tuve que aprender sola. (...) en primer año dedicaba la totalidad de mi tiempo al estudio (...) igual la Universidad me abrió la cabeza. (E-6; estudiante de 23 años, de tiempo completo, padres universitarios; se encuentra haciendo materias de $5^{\circ}$ año)

Entrar acá fue una cachetada... Hay una falta de relación entre el secundario y la vida universitaria. Me impactaba la cantidad de material de bibliografía, requería muchas horas de lectura. (...) Era alto el nivel de presión y las expectativas hacia los alumnos (...) Cuando volvi a desaprobar no pude soportar la tensión. (E-11; 24 años; abandona luego de dos años. Ingresa a Medicina, abandona nuevamente, e ingresa a la carrera terciaria de Enfermería en una institución privada; no trabaja; se mantiene con apoyo familiar; primera generación de universitarios)

Mi primer año fue un completo desastre. Aprobé dos materias. (E-3; 24 años; no trabaja, primera generación de universitarios; se encuentra haciendo materias de $3^{\circ}$ año)

Mucho costo para adaptarse, (...) primer año muy mal. Hay mucha distancia entre lo que pensaba que la facultad era y lo que era. (E-15; no trabaja; primera generación de universitarios; se encuentra haciendo materias de $3^{\circ}$ año)

Hay mucha diferencia en el material de primero con respecto a secundario. Volvería a poner el curso de ingreso con más exigencia, más herramientas. (E-22; 21 años; no trabaja, padres universitarios; se encuentra haciendo materias de $4^{\circ}$ año)

Así como en los fragmentos anteriores se señala el hiato entre contenidos y estrategias en el pasaje desde la educación secundaria a la universidad, para muchos de estos jóvenes de 
primera generación de universitarios, la educación superior representa un verdadero mundo que les resulta absolutamente ajeno.

El haber hecho un curso de ingreso hubiera cambiado bastante (...) mi primer año fue un completo desastre, me manejé absolutamente solo. (...) Es como que yo te diga a vos qué hacen, cómo viven los comisarios...ni idea debés tener, bueh a mí con la universidad me pasa lo mismo. (E-3; 24 años; no trabaja; primera generación de universitarios; ambos padres trabajan en servicio penitenciario; se encuentra haciendo materias de $3^{\circ}$ año)

Me di cuenta que transitar una carrera sola es casi imposible (...), no tenía a nadie para preguntarle (...), aprendí a moverme en la Universidad haciéndolo. (...) fui buscando por ensayo y error. (...) La Universidad es magia. (E-9; 21 años; no trabaja; primera generación de universitarios, proviene de otra ciudad, por lo que refiere mucho esfuerzo económico; se encuentra realizando materias de $3^{\circ}$ año)

En la transición del colegio a la facultad sin ninguna información previa de cómo es este nuevo ámbito, no sabía ni a donde ir. (...) aprendí a que hay que averiguar por nuestra cuenta. (E-13; 28 años, trabaja, primera generación universitarios; se encuentra realizando materias de $3^{\circ}$ año)

Surgen en algunos casos menciones sobre el uso y utilidad de otros dispositivos institucionales de apoyo a la inserción y afiliación durante el primer año. En general, los entrevistados que los utilizaron revalorizan la ayuda de los tutores pares y el Centro de Estudiantes. Así, sostienen:

(...) cuando no sabía dónde ir me decían en el centro de estudiantes. (E-1 1; 24 años; cambia de carrera luego de dos años; no trabaja; primera generación de universitarios)

Lo que más me sirvieron son las tutorías. (E-7; no trabaja; padres universitarios; se encuentra haciendo materias de 5 año)

[las tutorías] me sirvieron mucho en Biología. (E-12; 45 años; trabaja, tiene hijos; primera generación universitarios; se encuentra realizando materias de 2 y $3^{\circ}$ año)

...los del Centro de Estudiantes siempre te orientan. (E-21; 21 años; no trabaja; padres universitarios; se encuentra haciendo materias de 3 año)

...hay unos grupos de tutores pares, que me ayudó muchísimo. Son muy buena estrategia para bajar la ansiedad, especialmente en primer año. (E-16; 21 años; no trabaja; primera generación de universitarios; se encuentra realizando materias de $5^{\circ}$ año)

Es muy difícil organizar el tiempo personal (...) los tutores pares son de mucha ayuda. (E-19; trabaja 4 horas diarias; primera generación de universitarios; se encuentra haciendo materias de $3^{\circ}$ año)

En este sentido, muchos de los dispositivos de apoyo al ingresante son valorizados positivamente por los estudiantes. En particular, estudiantes avanzados, tutores pares y miembros del Centro de Estudiantes aparecen reiteradas veces como mecanismos que coadyuvaron a permanecer en la Facultad.

Otros entrevistados presentan opiniones negativas sobre la falta de ayuda desde la universidad misma y las dificultades consecuentes para poder adaptarse a la vida universitaria:

... mi hermana me explicaba algunas cosas, después nadie me ayudó, falta acompañamiento institucional. (E-5; 21 años; no trabaja; padre con estudios universitarios incompletos; tiene una hermana en la universidad; se encuentra realizando materias de $5^{\circ}$ año) 
... no tuve apoyo institucional en ninguna instancia (...) deberían enseñar a aprender, yo fui aprendiendo sola. (E-6; estudiante de 23 años; no trabaja; padres universitarios; se encuentra haciendo materias de $5^{\circ}$ año)

en primer año es muy difícil informarse, hay como una falta general de información. (E-13; 28 años; trabaja 8 horas diarias; primera generación universitarios; se encuentra realizando materias de $3^{\circ}$ año)

\subsection{Infraestructura y equipamiento}

Tanto los estudiantes que ingresaron antes como después del cambio de modalidad en el ingreso destacan como dificultades durante el primer año, cuestiones edilicias vinculadas a la imposibilidad del sistema de absorber tal cantidad de alumnos. El estado de las instalaciones (en especial los baños) y la relación entre el espacio y la cantidad de alumnos (cantidad de estudiantes por aula, tamaño de las aulas, falta de sillas) son tópicos recurrentes. Así expresan distintos entrevistados los problemas que creen más importantes respecto a la infraestructura y el equipamiento:

Caídas del techo (...) baños clausurados... cada dos por tres no hay cañón [proyector] o no tienen micrófono [los docentes]. (E-13; ingresante 2014; primera generación universitarios; se encuentra realizando materias de $3^{\circ}$ año)

Nulo mantenimiento y limpieza (...) aulas desbordadas de alumnos, los alumnos a veces se quedan sin poder cursar. (E-23; ingresante 2015; primera generación de universitarios; abandona la carrera en primer año)

Hay baños y equipamiento roto... (...) faltan sillas completas, algunas tienen roto el asiento o no tienen mesa para apoyar. (E-20; ingresante 2014; primera generación universitarios; se encuentra realizando materias de $4^{\circ}$ y $5^{\circ}$ año)

... la suciedad, la falta de docentes... los trámites, el maltrato de división alumnos, la desorganización general del sistema. (E-34; estudiante que abandona y decide estudiar Psicopedagogía en una universidad privada en la misma ciudad)

Es muy recurrente también entre las entrevistas la mención hacia la falta de espacio físico en la facultad. Si bien esto es algo que surge en distintos entrevistados, aparece más marcadamente en la cohorte 2015 , la primera en ingresar con modalidad irrestricta, luego del gran aumento en el número de ingresantes. Son estos estudiantes quienes más reportan haber sufrido situaciones como asistir a clases sentados en el piso o incluso tratando de escuchar parados en los pasillos, no escuchar a los docentes en las clases multitudinarias por la lejanía con respecto al mismo, etc.:

He cursado en el piso o sentado en la puerta del aula. (E-24; ingresante 2015; padres universitarios; se encuentra realizando materias de $4^{\circ}$ año)

He cursado en el piso, o en aulas hiper-llenas de las que no se podría salir en caso de haber una emergencia. (E-27, ingresante 2015; padres universitarios; se encuentra haciendo materias de $5^{\circ}$ año)

En varios teóricos o prácticos [clases teóricas o clases prácticas] no hay lugar suficiente. (E-26; ingresante 2015; se encuentra realizando materias de $3^{\circ}$ año)

...los primeros años de cursada fue peor. (E-28; ingresante 2015; padres universitarios; se encuentra realizando materias de $4^{\circ}$ y $5^{\circ}$ año)

Cursás afuera del salón, tenés que llegar antes para entrar. (E-19; ingresante 2015; trabaja 4 horas diarias; primera generación de universitarios; se encuentra haciendo materias de $3^{\circ}$ año)

Como que no hay un criterio lógico de cómo administrar las aulas. (E-9; ingresante 2015; primera generación de universitarios, se encuentra realizando materias de $3^{\circ}$ año) 
El edificio no cuenta con la cantidad de aulas necesarias para el número de alumnos. (E-11; ingresante 2014; abandona luego de dos años y actualmente estudia Enfermería en una institución privada; no trabaja; primera generación de universitarios)

Con la cantidad de alumnos que hay por aula, no se pueden apreciar las clases. (E14; ingresante 2014; abandona luego de tres años y actualmente estudia otra carrera en una universidad privada)

Desde el discurso de los estudiantes, se observa que las dificultades de espacio, que ya existían previamente, se acentúan con el incremento de la matrícula. La institución, saturada por la cantidad de alumnos y superada por déficits en infraestructura y equipamiento, no tuvo posibilidad de adaptar sus recursos (materiales y humanos), lo que generó efectos colaterales. Dado que las aulas existentes no están asignadas por años, las mismas se organizan y reparten en las franjas horarias según la demanda que exista. Al aumentar significativamente la demanda de las aulas para cubrir un mayor número de estudiantes de $1^{\circ}$ año, también se resintió la capacidad de cubrir la demanda de los años posteriores.

\subsection{Funcionamiento institucional y diseño curricular}

Ya fue mencionada la dificultad para adaptarse por la falta de ayuda de parte de la universidad. Aquí, aparecen ahora problemáticas sobre el funcionamiento de la institución en sí misma y de algunas oficinas en particular:

División alumnos funciona muy mal, tienen una mala atención al público, su forma de funcionar quedó obsoleta. Piden todo el papel y todo lleva muchos tiempos de espera. (E-3; 24 años; no trabaja, primera generación de universitarios; se encuentra haciendo materias de $3^{\circ}$ año)

División alumnos tiene una muy mala predisposición para atender, la inscripción [a las materias] funciona muy mal. (E-8; 22 años; primera generación de universitarios; ingresante 2015; no trabaja; se encuentra realizando materias de $3^{\circ}$ y $4^{\circ}$ año)

Asimismo, aparecen con alta prevalencia el desconocimiento y la ausencia de comprensión de lógicas implícitas y la necesidad de trabajar este tema. Los estudiantes aducen que, aunque se haya mencionado en el curso de ingreso, no llegan de ningún modo a poder evaluar los alcances que tendrá la demanda de la carga horaria, el impacto de las correlatividades de las materias y otras dimensiones similares. Así, expresan:

...cada profesor cree que su materia es la única o la más importante (...) no te explican la lógica de su materia en el plan de estudios. (E-4; 22 años; con sus hermanos son primera generación de universitarios; no trabaja; se encuentra finalizando sus estudios)

Distintos entrevistados presentan quejas específicas sobre la organización del plan de estudios, y algunos creen que el mismo debería organizarse y actualizarse. Reconocen que algunas materiales repiten el mismo material y que existe dogmatismo. en la formación:

Hay mucho material que habla de lo mismo y poca actualización. (E-22; 21 años; no trabaja, padres universitarios; se encuentra haciendo materias de $4^{\circ}$ año)

La cuatrimestralización lleva a que sean ridículos los tiempos en relación a los contenidos (...) es desproporcionada la relación tiempo - cantidad (...) llegás sin tener idea, (...) que te enseñen cómo se aprende en la Universidad. (E-4; 22 años; con sus hermanos son primera generación de universitarios; no trabaja; se encuentra finalizando sus estudios) 
los tiempos de cursada como cortos para la cantidad de contenidos que se dan (...) la organización actual no permite usar lo que se aprende. (E-6; estudiante de 23 años, de tiempo completo, padres universitarios; se encuentra haciendo materias de $5^{\mathrm{o}}$ año)

Preferiría un plan con más materias electivas, como en la UBA [Universidad de Buenos Aires], (...) mi primera forma de estudiar fue memorizar. (E-8; 22 años; primera generación de universitarios; ingresante 2015; no trabaja; se encuentra realizando materias de $3^{\circ}$ y $4^{\circ}$ año)

Hay mucho dogmatismo en la carrera. (E-3; 24 años; no trabaja, primera generación de universitarios; se encuentra haciendo materias de $3^{\circ}$ año)

Por lo general veo limitación en cuanto a las orientaciones... mucha prevalencia de psicoanálisis en las materias y en los textos. (E-17; trabaja y ha dejado la carrera de Psicología en suspenso por ahora, con solo el $20 \%$ de las materias aprobadas)

Hay materias que deberían darse con más tiempo. (E-1; estudiante de 32 años, trabaja parcialmente, segunda generación universitarios; se encuentra haciendo materias de $4^{\circ}$ año)

Hay muy poca carga de materias biológicas. (E-4; 22 años; con sus hermanos son primera generación de universitarios; no trabaja; se encuentra finalizando sus estudios)

...muchos materiales se repiten en distintas materias (...), deberían organizarse. (E2; estudiante, 43 años, a cargo de su padre, trabaja 4 horas al día, primera generación universitarios; se encuentra haciendo materia de $3^{\circ}$ año)

Muchos de los estudiantes, indistintamente de que hayan ingresado en el 2014 o en el 2015 , o de que están haciendo materias de $2^{\circ}$ o de $5^{\circ}$ año, se quejan de la falta de prácticas profesionales o la poca relación entre la formación y la profesión, principalmente refiriéndose al ámbito clínico. Hay críticas constantes a la falta de formación práctica, y la desvinculación entre la teoría que se enseña y la práctica profesional futura. Así, expresan:

todo desorganizado, (...) necesitamos más prácticas, las del futuro profesional (...) Me parece que está muy lejano el texto a lo que vamos a hacer en la práctica. Necesitamos más prácticas, estar más mano a mano con alguien, interacción humana. (E-3; 24 años; ingresante 2015; no trabaja; primera generación de universitarios; se encuentra haciendo materias de $3^{\circ}$ año)

La orientación (...) necesitaría más prácticas (...) abordaje de cuestiones prácticas. (E-7; ingresante 2015; no trabaja; padres universitarios; se encuentra haciendo materias de $5^{\circ}$ año)

...veo poca relación con las habilidades profesionales. (E-2; estudiante, 43 años, a cargo de su padre, trabaja 4 horas al día, primera generación universitarios; se encuentra haciendo materia de $3^{\circ}$ año)

Necesitamos más prácticas, está muy lejos lo que se ve en los textos de lo que se hace en la práctica. (E-3; ingresante 2015; 24 años; no trabaja, primera generación de universitarios; se encuentra haciendo materias de $3^{\circ}$ año)

En resumen, parece desprenderse que muchos estudiantes consideran necesaria una actualización en el funcionamiento institucional y en el diseño curricular, que sea más acorde con los tiempos actuales que corren. Demandan la actualización de los materiales de estudio para obtener herramientas claras para el abordaje profesional, y la organización de las pautas institucionales. También algunos entrevistados (E-17; E-18) hablan de la necesidad de digitalizar los materiales y del poco o nulo uso de la plataforma moodle. 


\subsection{Vínculos pedagógicos}

La interacción docente-alumno fue contemplada desde aspectos como la disponibilidad y actitud hacia la enseñanza, los recursos didácticos utilizados, el cumplimiento de las pautas y criterios de evaluación y las devoluciones respecto de los procesos de enseñanzaaprendizaje. Algunos entrevistados destacan la importancia de la formación y predisposición docente a la hora de aprender y cómo esto incide en el aprendizaje de los alumnos. Entre las entrevistas hay visiones favorables del cuerpo docente y otras desfavorables. Entre las consideraciones desfavorables más representativas, cabe señalar:

No hay pedagogía (...), nulo trabajo sobre la oralidad. (E-4; 22 años; ingresante 2014; con sus hermanos son primera generación de universitarios; no trabaja; se encuentra finalizando sus estudios)

... falta de capacitación en teoría de la enseñanza. (E-18; ingresante 2015; 22 años; no trabaja; padres universitarios; se encuentra realizando materias de 3 año)

siento que a la mayoría [de los docentes] le falta estrategias...creatividad. Como que no tienen capacidad para bajar los contenidos. (E-17; ingresante 2015; trabaja y ha dejado la carrera de Psicología en suspenso por ahora, con solo el 20\% de las materias aprobadas)

mayor contralor sobre el cuerpo de profesores, (...) falta de pasión. (E-27; ingresante 2015; padres universitarios; se encuentra haciendo materias de $5^{\circ}$ año)

Asistí a situaciones de humillación en un teórico de primer año cuando un alumno preguntaba lo que no entendía.... (E-19; ingresante 2015; trabaja 4 horas diarias; primera generación de universitarios; se encuentra haciendo materias de $3^{\circ}$ año)

... saben mucho [los docentes], pero no todos explican bien. (E-2; estudiant; 43 años, a cargo de su padre, trabaja 4 horas al día, primera generación universitarios; se encuentra haciendo materia de $3^{\circ}$ año)

yo diría hay dos tipos de docentes: el docente que te explica en que te equivocaste, en qué debes mejorar y el docente power point. (E-22; 21 años; no trabaja, padres universitarios; se encuentra haciendo materias de $4^{\circ}$ año)

Otros entrevistados, en cambio, tienen una visión más favorable del cuerpo docente. Entre las respuestas se destacan las siguientes:

la calidad humana. Estudiante que trabaja, ingresó con examen y proviene de familia de universitarios. (E-27, ingresante 2015; padres universitarios; se encuentra haciendo materias de $5^{\circ}$ año)

Hay docentes que hacen cosas positivas en primer año, por ejemplo, que los parciales tengan puntaje acumulativo (...) son los mismos que te preparan con tutorías. (E-2 1; 21 años; no trabaja; padres universitarios; se encuentra haciendo materias de 3 año)

Tengo una buena opinión de los aspectos docentes. (E-3; 24 años; no trabaja, primera generación de universitarios; se encuentra haciendo materias de $3^{\circ}$ año)

en general sentí una gran disponibilidad. (...) [Los docentes] tratan de responder de forma rápida a las consultas. (E-6; estudiante de 23 años, de tiempo completo, padres universitarios; se encuentra haciendo materias de $5^{\circ}$ año)

...la empatía de los docentes (...) son muy útiles las devoluciones que hacen en los parciales desaprobados y lo mismo en los finales. (E-16; 21 años; no trabaja; primera generación de universitarios; se encuentra haciendo materias de $5^{\circ}$ año) 
Algunos entrevistados (e.g., E-24; E-26) también refieren que existe mucha variabilidad en la calidad del cuerpo docente. La E-9 (21 años; primera generación de universitarios; cursa materias de $3^{\circ}$ año) refiere sobre el tema que "hay de todo, pero en general bien". Un entrevistado también asegura que hay mucha variabilidad y cuenta una experiencia negativa durante sus primeros años:

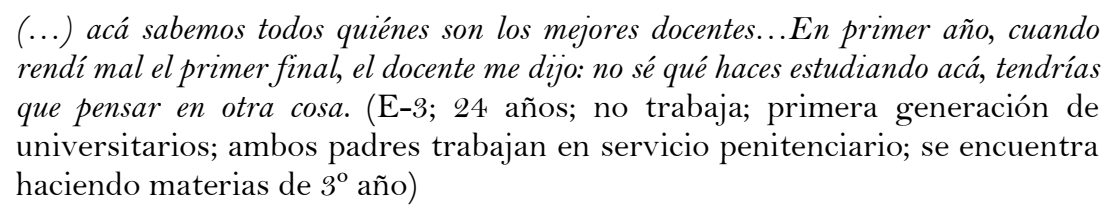

En general, en las entrevistas, se asiste a una tendencia general a marcar como áreas a mejorar en el cuerpo docente las dotes explicativas y la utilización de recursos didácticos, fundamentalmente la utilización de recursos y plataformas digitales. También que el docente incorpore las instancias de devolución de los exámenes como parte del proceso de enseñanza-aprendizaje, y la aproximación al futuro rol profesional. En la mayor parte de los casos, además de los defectos, se mencionan buenas experiencias y se valoran los esfuerzos específicos de algunos profesores.

\subsection{Grupo de pares}

Acerca de los grupos de pares, en aquellos entrevistados que se explayaron respecto de los motivos de su sensación de dificultad en la inserción social en la facultad, el grupo de pares es recurrentemente citado como fuente de acompañamiento; desde formas sistematizadas como los Tutores pares o el Centro de estudiantes, o desde el lugar de compañero que está atravesando una situación y vivencia similar.

Mis compañeros, (...) tenerlos me ayudó, por ejemplo, en mis aprendizajes. (E-16; 21 años; no trabaja; primera generación de universitarios; se encuentra realizando materias de $5^{\circ}$ año)

Mi adaptación al grupo de estudiantes en general fue bastante buena; hay mucha apertura a establecer vínculos nuevos con los demás. (E-3; 24 años; no trabaja, primera generación de universitarios; se encuentra haciendo materias de $3^{\circ}$ año)

Siempre senti a todos muy sociales, tuve una excelente experiencia en ese aspecto. (E4; 22 años; con sus hermanos son primera generación de universitarios; no trabaja; se encuentra finalizando sus estudios)

Como contracara de esto, están aquellos casos donde el entorno de pares se vivenció como hermético u hostil, haciendo más solitaria la experiencia de inserción a la vida universitaria:

[Un entorno humano] como muy competitivo y egoísta. (E-2; estudiante, 43 años, a cargo de su padre, trabaja 4 horas al día, primera generación universitarios; se encuentra haciendo materia de $3^{\circ}$ año)

Poco compañerismo en general, y egoísmo. (E-6; estudiante de 23 años, de tiempo completo, padres universitarios; se encuentra haciendo materias de $5^{\circ}$ año)

Aunque no de manera exclusiva, esta apreciación sobre un ambiente social hermético es mencionada por las tres personas entrevistadas que se trasladaron desde otras localidades para estudiar en esta universidad:

El clima dependía del grupo que te tocara, según la clase social... (E-1; estudiante de 32 años; trabaja parcialmente, segunda generación universitarios; se encuentra haciendo materias de $4^{\circ}$ año; se traslada a Mar del Plata desde una localidad pequeña de 60.000 habitantes a 250 kilómetros). 
Me sentí excluida. Gente falsa (...) incómoda. No conocía a nadie. (...) Los primeros años fue horrible. Hubiera desertado si el grupo siguiera siendo el inicial. (E-9; 21 años; no trabaja; primera generación de universitarios, proviene de otra ciudad, por lo que refiere mucho esfuerzo económico; se encuentra realizando materias de $3^{\circ}$ año; se traslada a Mar del Plata desde una localidad pequeña de 1.000 habitantes a 45 kilómetros)

Tengo un buen grupo de pares, pero en general somos todos de afuera de la ciudad. La gente de acá es diferente. (E-8; 22 años; primera generación de universitarios; ingresante 2015; no trabaja; se encuentra realizando materias de $3^{\circ}$ y $4^{\circ}$ año; se traslada a Mar del Plata desde una localidad de 110.000 habitantes a 300 kilómetros)

Similar a lo observado en el caso de las experiencias sobre la calidad docentes, existe una amplia variabilidad en las experiencias con el grupo de pares. Existe consenso en la importancia de atravesar la formación universitaria acompañada de personas que se encuentren en la misma situación.

\section{Discusión y conclusiones}

El presente estudio tuvo por objetivo explorar las percepciones de los estudiantes universitarios sobre sus propias trayectorias educativas en el marco de la implementación de las políticas de ingreso irrestricto, y en relación con factores sociales, económicos e institucionales. En primer lugar, se observa que las variables socioeconómicas siguen siendo un obstáculo en el acceso y la permanencia, lo cual es similar a lo reportado por Coschiza y otros (2016) y Fiori y Ramírez (2013), y a lo sugerido por autores como Ezcurra (2011, 2019), sobre el acceso real de los estudiantes que pertenecen a sectores sociales más desfavorecidos. A pesar de que la universidad sea gratuita, los estudiantes cuyos padres no pueden costear su mantenimiento (vivienda, alimentación, material educativo) se ven obligado a trabajar y, por lo tanto, a dividir sus tiempos entre la universidad de la vida laboral. En este sentido, resulta muy difícil sostener el tiempo académico de cinco años de duración de la carrera, llevando, en el mejor de los casos, a una lentificación o rezago y, en otros casos, a la deserción (García de Fanelli, 2014; González et al., 2020).

Sobre el género, unas pocas estudiantes mujeres que eran madres reconocieron la dificultad para coordinar la vida familiar con los tiempos académicos. Sin embargo, solo una de ellas ha abandonado definitivamente la carrera para atender a las tareas que históricamente se les demandan a las mujeres en el ámbito doméstico. En cambio, entre los entrevistados hombres, dos de ellos abandonaron la carrera para poder salir al mercado laboral, lo cual, si se tiene en cuenta la proporción de hombres, representan el 25\% de los entrevistados. Aunque estos números no son representativos en términos estadísticos, sí son llamativos en términos cualitativos, y se alinean con lo sugerido ya en la literatura previa (e.g. Fiori y Ramírez, 2013) sobre la existencia de un mayor riesgo de deserción entre los hombres que entre las mujeres.

Sobre la adaptación al funcionamiento institucional, los entrevistados describen el momento de transición entre la educación secundaria y la universitaria como un tránsito entre dos lógicas opuestas. Autores como Fernández y Carlino (2010) destacan las discrepancias entre las lógicas implícitas de la educación secundaria y la universitaria. La inexistencia de conocimientos previos, el desconocimiento de los términos específicos de la disciplina, sumado a la falta de herramientas de trabajo y de técnicas de estudio, 
complejiza aún más la tarea de aprendizaje. Es allí cuando los entrevistados destacan la importancia y necesidad de una alfabetización académica, a través de la revalorización de las herramientas o dispositivos de apoyo a los ingresantes: el curso de ingreso (restrictivo o no), los dispositivos de tutorías para preparar exámenes y el sistema de tutores pares. Similarmente, también Mendoza y Flores (2007) indican que los estudiantes desertan menos cuando son acompañados en su trayectoria educativa por medio de dispositivos de apoyo.

Sobre los recursos de acompañamiento, los mismos parecen suponer un espacio donde los aspirantes pueden reflexionar sobre esta transición y trabajar la adaptación. En este sentido, alfabetizar académicamente es enseñar a leer y escribir en otro idioma, el de la disciplina (Massone y González, 2005; Perrenoud, 2006). Los ingresantes debieran ser fuertemente apoyados por mecanismos institucionales, y con especial énfasis en el caso de aquellos estudiantes que son primera generación de universitarios, dado que este grupo aparece como el que denota mayor extrañamiento con respecto a la Universidad. Similar a la propuesta de Tinto (2012), la implementación de estrategias proactivas y estructuradas por parte de la Universidad es determinante para el real acceso de los estudiantes desventajados. Un abordaje institucional durante el primer año de estudios (Ezcurra, 2011) debe centrar la atención en prácticas de apoyo a los estudiantes para andamiar, acompañar y dar sostén en los primeros tramos de su trayectoria en términos de tutorías académicas, cursos de nivelación y perfeccionamiento docente.

En este contexto, también muchos estudiantes también mencionan la importancia de contar con buenos docentes en su trayectoria educativa, lo cual es similar a lo reportado por Márquez García y Padua Arcos (2009) sobre la importancia de la cercanía y el afecto de los docentes. Las entrevistas aquí reportadas, también sugieren una gran variabilidad en la calidad de los docentes, lo cual también ha sido puesto de manifiesto en estudios previos (p. ej.,. Aisenson et al., 2011).

Además de los aspectos más estrictamente académicos, los entrevistados destacan la necesidad de mayor apoyo institucional en la adaptación a la vida universitaria, sobre todo respecto a aspectos burocráticos o de funcionamiento, como la ubicación de las aulas, los trámites de matriculación, la facilitación de las inscripciones a las materias, o la mejora en el trato de parte de las personas que trabajan en las oficinas administrativas. Muchos manifiestan haber aprendido solos. Otros refieren la importancia de contar con la ayuda de los pares y compañeros en este proceso, lo cual es compatible con lo reportado por Cardozo-Ortiz (2011) sobre la utilidad del tutor-par en la prevención de la deserción. Debe considerarse que, por fuera de las normas explícitas, las universidades poseen una serie de reglamentaciones y normas que pueden considerarse implícitas y que son desconocidas por los que ingresaron recientemente a la institución (Marcillo, 2019). El currículo debe ser entendido como un conjunto de principios que validan la enseñanza y el desconocimiento de los estudiantes, y los problemas que describen los estudiantes para recorrerlo supone un verdadero desafío a ser resuelto institucionalmente. La situación es aún más compleja para aquellos ingresantes que representan la primera generación de universitarios en su familia, pues no solo desconocen los recursos, sino también las canales de acceso a la información que necesitan (Ezcurra, 2011).

También, los resultados indican que la facultad ha tenido dificultades para adaptarse el creciente número de estudiantes generado por las políticas de ingreso irrestricto, entre otras medidas de masificación (López Segrera, 2016). Según Rama (2009), esta 
masificación produce en sí misma cambios en el perfil social del estudiantado, incorporando ahora estudiantes con características diferentes a las que tenían históricamente los estudiantes (Brunner, 2012). Los hallazgos coindicen con lo propuesto por Fernández Lamarra y Costa de Paula (2002), quienes aseveran que las dificultades son, tanto de los nuevos públicos para adaptarse a la universidad, como de la universidad misma para adaptarse a estos nuevos perfiles y crear mecanismos de acceso, recepción y permanencia. En particular, la relación entre el espacio áulico y la cantidad de alumnos aparece recurrentemente como un problema, dejando claro la incapacidad de la institución para absorber tal cantidad de estudiantes.

En general, los hallazgos suponen un aporte a la interpelación del denominado "ingreso directo" como único mecanismo de intervención institucional para coadyuvar al acceso y permanencia de los nuevos públicos que ingresan a la educación superior (Tinto, 2012). La verdadera democratización del acceso inicia por la igualdad en el ingreso, pero debe avanzar hacia el trayecto educativo más general (Bolívar, 2012; Cuenca, 2012) y hacia la flexibilización de la estructura pedagógica tradicional (Di Piero y Mataluna, 2018; González y del Valle, 2020). La Universidad requiere una política institucional que abarque la organización de forma comprehensiva y coordinada, y abordaje que implique a la institución toda, incluso la adecuación de su infraestructura frente a la nueva demanda. Esto es, más allá de que los estudiantes puedan disponer de dispositivos de apoyo, la exigencia es contar con un compromiso institucional de naturaleza sustantiva con respecto a la enseñanza y el aprendizaje en busca de una inclusión no excluyente (Ezcurra, 2019). Si la educación superior atiende al ingreso de los estudiantes, pero no a su permanencia, se corre el riesgo de producir un efecto de puerta giratoria (Tinto, 2012). Es fundamental instrumentar acciones que coadyuven a la permanencia y regularidad en los estudios y a evitar el abandono: que las brechas de graduación no supongan brechas sociales.

\section{Referencias}

Aisenson, D., Virgili, N., Siniuk, D., Polastri, G., Rivarola, R., Rivero, L. y Schwarcz, J. (2011). Construcción de la trayectoria educativa: El inicio del ciclo secundario, ¿inserción o exclusión?, ¿'oportunidad o reproducción? Anuario de Investigaciones, 18, 133-141.

Bolívar, A. (2012). Justicia social y equidad escolar. Una revisión actual. Revista Internacional de Educación para la Justicia Social, 1(1), 9-45.

Bourdieu, P. (2005). Capital cultural, escuela y espacio social. Siglo XXI.

Brunner, J. J. (2012). La idea de universidad en tiempos de masificación. Revista Iberoamericana de Educación Superior, 3(7), 130-145. https://doi.org/10.22201/iisue.20072872e.2012.7.69

Cardozo-Ortiz, C. E. (2011). Tutoría entre pares como una estrategia pedagógica universitaria. Educación y Educadores, 14(2), 309-325. https://doi.org/10.5294/edu.2011.14.2.4

Carlino, P. (2004). La distancia que separa la evaluación escrita frecuente de la deseable. Universidad de Los Andes.

Carlino, P. (2005). Escribir, leer y aprender en la universidad: una introducción a la alfabetización académica. Fondo de Cultura Económica.

Chiroleu, A. (2009a). La inclusión en la educación superior como política pública: tres experiencias en América Latina. Revista Iberoamericana de Educación, 48(5), 1-15.

https://doi.org/10.35362/rie4852153 
Chiroleu, A. (2009b). Políticas públicas de inclusión en la educación superior los casos de Argentina y Brasil. Pro-Posições, 20(2), 141-166. https://doi.org/10.1590/So103-73072009000200010

Coschiza, C. C., Fernández, J. M., Redcozub, G. G., Nievas, M. E. y Ruiz, H. E. (2016). Características socioeconómicas y rendimiento académico. El caso de una universidad argentina. REICE. Revista Iberoamericana sobre Calidad, Eficacia y Cambio en Educación, 14(3), 51-76. https://doi.org/10.15366/reice2016.14.3.003

Cuenca, R. (2012). Sobre justicia social y su relación con la educación en tiempos de desigualdad. Revista Internacional de Educación para la Justicia Social, 1(1), 79-93.

Di Piero, E. y Mataluna, M. (2018). Educación secundaria en instituciones dependientes de universidades públicas: miradas docentes que consolidan su prestigio en Brasil y Argentina. Trabajo y Sociedad, 30, 391-410.

Ezcurra, A. M. (2011). Masificación y enseñanza superior: una inclusión excluyente. En N. Fernández Lamarra y M. F. Costa de Paula (Comps.), La democratización de la educación superior en América Latina (pp. 60-72). EDUNTREF.

Ezcurra, A. M. (2019). Derecho a la educación. Expansión y desigualdad: Tendencias y políticas en Argentina y América Latina. UNTREF.

Fernández, G. y Carlino, P. (2010). ¿En qué se diferencian las prácticas de lectura y escritura de la universidad y las de la escuela secundaria? Lectura y Vida, 31(3), 6-19.

Fernández Lamarra, N. y Costa de Paula, M. F. (2002). La democratización de la educación superior en América Latina. EDUNTREF.

Fiori, N. y Ramírez, R. (2013). Análisis de las trayectorias y perfil de los estudiantes desafiliados en la Universidad de la República en el período 2007-2012. En III Conferencias Latinoamericana sobre el Abandono en la Educación Superior. Ciudad de México, México.

García de Fanelli, A. M. (2014). Rendimiento académico y abandono universitario: Modelos, resultados y alcances de la producción académica en la Argentina. Revista Argentina de Educación Superior, 6(8), 9-38.

González, G. I. y del Valle, M. (2020). Estudio comparativo de trayectorias educativas en un colegio preuniversitario argentino según modalidad de ingreso: el caso del ingreso directo. Propuesta Educativa, 52(2), 180-193.

González, G. I., del Valle, M, Nogueira, F. y Grossi, C. (2020). Del examen selectivo al ingreso irrestricto. Estudio comparativo del desempeño académico según la modalidad de ingreso en una universidad nacional argentina. ¿Democratización o masificación? Praxis Educativa, 24(2), 1-15. https://doi.org/10.19137/praxiseducativa-2020-240207

Guadagni, A., Lima, G. y Boero, F. (2019). Tenemos más estudiantes universitarios, pero menos graduados que Brasil y Chile. Centro de Estudios de la Educación Argentina, 8(80), art 4.

Klein, F. (2011). Las tensiones en la relación docente-alumno. Una investigación del ámbito educativo. Aposta. Revista de Ciencias Sociales, 51, 1-28.

Latapí Sarre, P. (2012). Educación y justicia social. Revista Internacional de Educación para la Justicia Social, 1(1),199-202.

López Segrera, F. (2016). Educación superior comparada: Tendencias mundiales y de América Latina y Caribe. Revista de Avaliacao Superior, 21(1), 13-32. https://doi.org/10.1590/S1414-40772016000100002

Marcillo, C. (2019). ¿Qué es el currículum oculto? Revista Científica Retos de la Ciencia, 3(1), 58-66. 
Márquez García, M. J. y Padua Arcos, D. (2009). La institución educativa, un espacio a revisar: Las adolescentes gitanas en su trayectoria educativa. Revista Interuniversitaria de Formación del Profesorado, 23(1), 73-88.

Massone, A. y Gonzalez, G. (2005). Lectura: comprensión vs. retención de información. Una interpretación cognitiva. Revista Iberoamericana de Educación, 36(13), 11-17. https://doi.org/10.35362/rie36133048

Mataluna, M. (2018). Expectativas de los ingresantes a primer año en colegios de nivel medio en Argentina y Brasil: un estudio comparado en instituciones dependientes de Universidades Públicas. En B. Buenaventura, J. Del Cueto, E. Di Piero, C. Parellada y J. Pérez Zorrilla (Comps.), Nuevos desafíos en educación: Una mirada interdisciplinaria (pp. 59-70). FLACSO.

Mendoza, A. M. y Flores, S. M. (2007). Efectividad de un programa de apoyo educativo sobre la trayectoria académica de alumnos de licenciatura. Revista Mexicana de Psicología, 24(2), 243257.

Mollis, M. (2008). Las reformas de la educación superior en argentina para el nuevo milenio. Avaliação: Revista da Avaliação da Educação Superior, 13(2), 509-532.

https://doi.org/10.1590/S1414-40772008000200012

Montes, N. y Sendón, M. A. (2006). Trayectorias educativas de estudiantes de nivel medio. Argentina a comienzos del siglo XXI. Revista Mexicana de Investigación Educativa, 1 1(29), 381-402.

Morduchowicz, A. (2003). Discursos de economía de la educación. Losada.

Parrino, M. C. (enero, 2010). Deserción en el primer año universitario. Dificultades y logros. Comunicación presentada en el X Coloquio Internacional sobre Gestión Universitaria en América del Sur. Mar del Plata, Argentina.

Perrenoud, P. (2006). El oficio de ser alumno y el sentido del trabajo escolar. Popular.

Porto, A., Di Gresia, L. y López, M. (marzo, 2004). Mecanismos de admisión a la universidad y rendimiento de los estudiantes. XXXIX Reunión Anual de la AAEP Universidad Católica Argentina. Buenos Aires, Argentina.

Rabossi, M. (2014). Acceso (¿ingreso?) a las universidades nacionales argentinas: permisividad y consecuencias. Páginas de Educación, 7(2), 81-103. https://doi.org/10.22235/pe.v7i2.507

Rama, C. (2006). La tercera reforma de la educación superior en América Latina y el Caribe: Masificación, regulaciones e internacionalización. Revista Educación y Pedagogía, 18(46), 11 24.

Rama, C. (2009). La tendencia a la masificación de la cobertura de la educación superior en América Latina. Revista Iberoamericana de Educación, 50,173-195. https://doi.org/10.35362/rie500668

Rawls, J. (1971). A theory of justice. Oxford University Press.

Tinto, V. (2006). Research and practice of student retention: What next? Journal of College Student Retention: Research, Theory \& Practice, 8(1), 1-19. https://doi.org/10.2190/4YNU-4TMB-22DJ-AN4W

Tinto, V. (2012). Completing college: Rethinking institutional action. University of Chicago Press. https://doi.org/10.7208/chicago/9780226804545.001.0001

Tiramonti, G. y Ziegler, S. (2008). La educación de las élites. Aspiraciones, estrategias y oportunidades. Paidós. 
Ziegler, S. (2014), La escuela secundaria: Principales temas y problemas en perspectiva latinoamericana FLACSO.

\section{Breve CV de las autoras}

\section{Gloria González}

Licenciada en Ciencias de la Educación, Diplomada Superior en Ciencias Sociales y Educación y doctoranda en Política y Gestión de la Educación Superior. Ha sido Subsecretaria de Gestión Educativa de la Universidad Nacional de Mar del Plata y ha trabajado como responsable del programa Calidad Educativa en la Secretaría de Planeamiento de la misma Universidad. Es docente de las cátedras de Teorías del Aprendizaje y Psicología Cognitiva. Es directora del Grupo Investigación en Alfabetización Académica, y su principal línea de investigación ha sido la interacción entre vulnerabilidad socioeducativa y educación formal (en todos los niveles del sistema educativo). Posee numerosas presentaciones a congresos nacionales e internacionales. Es co-responsable de los textos sobre Teorías del Aprendizaje, la capacitación docente de la Dirección General de Escuelas y de los textos destinados al Ingreso a la Universidad. ORCID ID: https://orcid.org/0000-0001-7582-7908. Email: gigon@mdp.edu.ar

\section{Florencia Nogueira}

Licenciada en Psicología por la Universidad Nacional de Mar del Plata -UNMDP-. Especialista en docencia universitaria. Se desempeña como docente de la cátedra de Neuropsicología y participa desde el año 1993 de grupos de investigación en dicha facultad. Formada en neurociencias, posee publicaciones internacionales en el área. También se ha desempeñado en grupos de extensión relativos a la articulación entre neurociencias y educación. Actualmente es investigadora en el grupo de Alfabetización académica. ORCID ID: https://orcid.org/0000-0002-8392-9290. Email: fnogueir@mdp.edu.ar

\section{Macarena Verónica del Valle}

Licenciada en Psicología por la Universidad Nacional de Mar del Plata. También realizó una Especialización en Psicoterapia Cognitiva en la Fundación Aiglé y un Master en Investigación en Educación en la Universidad de Valladolid, España. Actualmente, es estudiante del Doctorado en Psicología de la UNMDP y Becaria Doctoral del CONICET. Es docente en la cátedra de Teorías del Aprendizaje de la UNMDP y auxiliar en proyectos de investigación del Instituto de Psicología Básica, Aplicada y Tecnología -IPSIBAT-. Macarena ha asistido a diversos cursos de posgrado y ha sido expositora en congresos de alcance nacional e internacional sobre procesos básicos, aprendizaje, funciones ejecutivas, características psicológicas, psicología cognitiva y metodología de investigación, medición y evaluación. Se área de trabajo son los procesos de autorregulación cognitiva y emocional en el ámbito educativo. ORCID ID: https://orcid.org/0000-0003-3549-7224. Email: mdelvalle@conicet.gov.ar

\section{María Carolina Grossi}

Licenciada en Psicología por la Universidad Nacional de Mar del Plata. En el área asistencial, trabaja como psicoterapeuta en consultorio privado. Es miembro del Grupo 
Alfabetización Académica, Profesora Titular en la Cátedra de Neuropsicología, y docente en la Cátedra Psicología Cognitiva. Se desempeña como Coordinadora del Ingreso a Psicología y ha realizado y documentado intervenciones destinadas a mejorar el rendimiento académico de ingresantes y estudiantes. Ha asistido a cursos de posgrado y ha sido expositora en congresos de alcance nacional e internacional, y ha posee producción sobre paradojas pragmáticas y sus efectos en las interacciones humanas, y sobre la referencia de los pronombres personales en su relación con el psiquismo. ORCID ID: https://orcid.org/0000-0002-7510-3210.Email: margros@mdp.edu.ar 